\title{
Aggrecanase degradation of type III collagen is associated with clinical knee pain
}

Bay-Jensen, A. C.; Kjelgaard-Petersen, C. F.; Petersen, K. K.; Arendt-Nielsen, L.; Quasnichka, H. L.; Mobasheri, A.; Karsdal, M. A.; Leeming, D. J.

Published in:

Clinical Biochemistry

Link to article, DOI:

10.1016/j.clinbiochem.2018.04.022

Publication date:

2018

Document Version

Peer reviewed version

Link back to DTU Orbit

Citation (APA):

Bay-Jensen, A. C., Kjelgaard-Petersen, C. F., Petersen, K. K., Arendt-Nielsen, L., Quasnichka, H. L., Mobasheri, A., Karsdal, M. A., \& Leeming, D. J. (2018). Aggrecanase degradation of type Ill collagen is associated with clinical knee pain. Clinical Biochemistry, 58, 37-43. https://doi.org/10.1016/j.clinbiochem.2018.04.022

\section{General rights}

Copyright and moral rights for the publications made accessible in the public portal are retained by the authors and/or other copyright owners and it is a condition of accessing publications that users recognise and abide by the legal requirements associated with these rights.

- Users may download and print one copy of any publication from the public portal for the purpose of private study or research.

- You may not further distribute the material or use it for any profit-making activity or commercial gain

- You may freely distribute the URL identifying the publication in the public portal 


\section{Accepted Manuscript}

Aggrecanase degradation of type III collagen is associated with clinical knee pain

A.C. Bay-Jensen, C.F. Kjelgaard-Petersen, K.K. Petersen, L. Arendt-Nielsen, H.L. Quasnichka, A. Mobasheri, M.A. Karsdal, D.J. Leeming

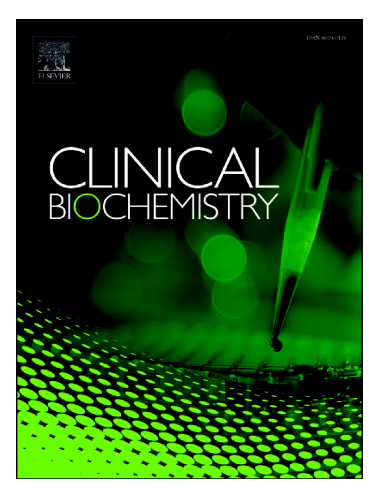

PII:

S0009-9120(17)30788-9

DOI: doi:10.1016/j.clinbiochem.2018.04.022

Reference: CLB 9774

To appear in:

Clinical Biochemistry

Received date:

4 August 2017

Revised date: 17 April 2018

Accepted date: 23 April 2018

Please cite this article as: A.C. Bay-Jensen, C.F. Kjelgaard-Petersen, K.K. Petersen, L. Arendt-Nielsen, H.L. Quasnichka, A. Mobasheri, M.A. Karsdal, D.J. Leeming , Aggrecanase degradation of type III collagen is associated with clinical knee pain. The address for the corresponding author was captured as affiliation for all authors. Please check if appropriate. $\mathrm{Clb}(2018)$, doi:10.1016/j.clinbiochem.2018.04.022

This is a PDF file of an unedited manuscript that has been accepted for publication. As a service to our customers we are providing this early version of the manuscript. The manuscript will undergo copyediting, typesetting, and review of the resulting proof before it is published in its final form. Please note that during the production process errors may be discovered which could affect the content, and all legal disclaimers that apply to the journal pertain. 


\section{Aggrecanase degradation of type III collagen is}

\section{associated with clinical knee pain}

AC Bay-Jensen ${ }^{1}$, CF Kjelgaard-Petersen ${ }^{1,2}$, KK Petersen ${ }^{3}$, L Arendt-Nielsen ${ }^{3}$, HL Quasnichka ${ }^{4,6}$ A. Mobasheri $^{4,5,6,7,8}$, MA Karsdal ${ }^{1,9}$, DJ Leeming ${ }^{9}$

${ }^{1}$ Rheumatology, Biomarkers and Research, Nordic Bioscience, Herlev, Denmark

${ }^{2}$ Department of Biotechnology and Biomedicine, Technical University of Denmark, Kgs. Lyngby, Denmark

${ }^{3}$ Center for Sensory-Motor Interaction (SMI), Department of Health Science and Technology, Faculty of Medicine, Aalborg University, Denmark

${ }^{4}$ The D-BOARD European Consortium for Biomarker Discovery

${ }^{5}$ Department of Veterinary Pre-Clinical Sciences, School of Veterinary Medicine, University of Surrey, Guildford, GU2 7AL, United Kingdom

${ }^{6}$ Faculty of Health and Medical Sciences, Duke of Kent Building, University of Surrey, Guildford, Surrey, GU2 7XH, United Kingdom

${ }^{7}$ Arthritis Research UK Centre for Sport, Exercise and Osteoarthritis, Queen's Medical Centre, Nottingham, NG7 2UH, United Kingdom

${ }^{8}$ Center of Excellence in Genomic Medicine Research (CEGMR), King Fahd Medical Research Center (KFMRC), Faculty of Applied Medical Sciences, King Abdulaziz University, Jeddah, 21589, Kingdom of Saudi Arabia

${ }^{9}$ Fibrosis Biology, Biomarkers and Research, Nordic Bioscience, Herlev, Denmark

Corresponding author: Anne C. Bay-Jensen, Nordic Bioscience A/S, Herlev Hovedgade 207, DK-2730 Herlev, Denmark. Tel. +45 44525216 Fax: +45 44525251 E-mail: acbj@nordicbio.com 


\section{ABSTRACT}

There is a lack of biochemical markers for non-invasive and objective assessment of symptomatic osteoarthritis (OA). Aggrecanase activity has been shown to be associated with joint deterioration and symptomatic disease through the degradation of extracellular matrix proteins, such as type III collagen. Our study aimed to identify and develop a novel biomarker by measuring an aggrecanase-mediated type III collagen neoepitope, and correlate levels of this biomarker with OA joint pain. Mass spectrometric analysis of purified type III collagen, degraded by the aggrecanase A Disintigrin and Metalloproteinase with Thrombospondin motif (ADAMTS), revealed a fragment generated by ADAMTS-1, -4 and -8 . A monoclonal antibody was raised against the neoepitope of this fragment (COL3-ADAMTS) and a competitive ELISA was developed and tested; using serum samples from a cross-sectional cohort of patients with different degrees of knee OA $(n=261)$. The COL3/ADAMTS ELISA was technically robust and specific for the ADAMTS-1, -4 and -8 generated neoepitope. COL3/ADAMTS was released form cytokine stimulated synovial cultures, indicating a biologic link between the marker and synovium. In OA patients, serum COL3/ADAMTS was independently associated with pain scores (rho=-0.13-0.17, $p<0.05$ ). This association was associated significantly with the presence of radiographic OA. Together, these data indicate that COL3/ADAMTS could be a marker of early osteoarthritis and the underlining pathology.

Keywords: Biochemical markers, type III collagen, pain, OA, ADAMTS, protease-cleaved neoepitope.

\section{Keywords: Type III collagen, ADAMTS, Knee osteoarthritis, neoepitope, biochemical marker}




\section{ABBRIVIATIONS}

ADAMTS A Disintigrin and Metalloproteinase with Thrombospondin motif

COL3 type III collagen

ECM extracellular matrix

OA osteoarthritis

VAS visual analog score 


\section{HIGHLIGHTS}

- That a technical robust ELISA, COL3/ADAMTS, was developed which show good sensitivity for measurement in human serum samples

- COL3/ADAMTS was released from human osteoarthritis synovial membranes cultured is the presence of proinflammatory cytokines, indicating a link to synovial pathology and inflammation

- When the marker was measured in serum of 261 subjects with or without knee pain, we found that it was significantly and inversely associated with radiographic knee osteoarthritis. 


\section{INTRODUCTION}

Osteoarthritis (OA) is characterized by a gradual deterioration of articular joint cartilage, along with remodeling of subchondral bone and synovial inflammation. The process is partly driven by an upregulation of aggrecanases and matrix metalloproteinases (MMPs), which degrade and remodel the extracellular matrix (ECM) of joint tissues [1]. The ECM mainly consists of collagens, proteoglycans and glycoproteins, all of which play important and unique functions in maintaining the physicochemical structure of tissue [1-3]. ECM remodelling is a normal process in which formation and degradation of healthy tissue is balanced, however an imbalance exists in disease, which manifests itself in various pathologies. Degradation of the cartilage by the aggrecanase, A Disintigrin and Metalloproteinase with Thrombospondin motif (ADAMTS), is well described [2][3]; whereas the role of ADAMTS in the turnover of the fibrous synovial tissue, is less so. Increased ADAMTS activity has been found in the synovial fluid of OA patients[4], thus the action of these enzymes may not only be restricted to the cartilage.

The fibril-forming type III collagen is mainly associated with type I collagen, and together they provide the structural framework of most connective tissues, including the synovial membrane which surrounds synovial joints [5]. Type III collagen contributes to synovial tissue elasticity, a property that is uniquely connected to this type of collagen [6]. Type III collagen is also found in healthy cartilage and is thought to stabilize the type II collagen framework [7].

The pathogenesis of OA involves an inflammatory response, and the activation of chondrocytes, fibroblasts and leukocytes partly mediates tissue turnover by the secretion of MMPs and ADAMTSs [3]. Type III collagen is upregulated in response to pro-inflammatory cytokines such as tumor necrosis factor $\alpha$ (TNF- $\alpha)$ and interleukin $1 \beta$ (IL-1 $\beta$ )[8] which results in thickening of the synovial membrane. In addition, type III collagen has been found to be elevated in osteoarthritic cartilage [9]. Pro-inflammatory cytokines also upregulate the release of ADAMTS from articular cartilage leading to degradation of both cartilage and synovial membrane ECM $[10,11]$. Collagen fragments are released into circulation and can potentially be assessed as markers of joint degradation in synovial fluid, serum and urine. Such protein fragments, referred to as neoepitopes or protein fingerprints $[12,13]$, have proven to be more accurate than their intact protein of origin, in detecting and quantifying certain pathophysiological processes [14]. For example, fragments of type II collagen, generated by MMPs, have been shown to be markers of cartilage degradation and joint deterioration in OA and rheumatoid arthritis (RA) [15]. 
The aim of the present study was to develop a novel ELISA specific for an ADAMTS generated fragment of type III collagen. In addition, we determined whether ADAMTS mediated degradation of type III collagen may be useful for monitoring joint type III collagen turnover in OA, along with its relationship with symptomatic disease, particularly pain and function.

\section{MATERIALS AND METHODS}

\subsection{Patient samples}

Serum COL3/ADAMTS levels were measured in a total of 261 subjects belonging to the cross-sectional C4P-003 study [16]. Subjects had different degrees of primary knee OA defined by different degrees of knee pain (maximal pain during the last 24 hours rated on a visual analog scale [VAS]) (VAS 0-100), and different degrees of radiological knee OA (Kellgren and Lawrence grade (KLG)). Radiographic knee OA (RKOA) was defined as having a KLG of 2 [17]. Only subjects which had a complete set of matching serum, X-rays and questionnaires available were included in the current investigation (261 out of 282 subjects with available serum samples)[18]. Subjects were segregated into four groups based on their VAS pain score: no pain: VAS <10 $(n=52)$, mild pain: VAS 10 to $39(n=81)$, moderate pain: VAS 40 to 69 ( $n=67)$ and severe pain: VAS 70 to $100(n=61)$. Age, BMI, and disease duration (if any), as well as WOMAC [19] and Lequesne [20] scores were collected (table 1). All subjects were Caucasian and abstained from analgesic medication for at least 72 hours [16]. The study was approved by the Danish ethical committee (VEK; The Ethical committee of North Jutland no.: N-20100094).

\subsection{Reagents}

All reagents used for experiments were standard high-quality chemicals from companies such as Merck (Whitehouse Station, NJ, USA) and Sigma Aldrich (St. Louis, MO, USA). The synthetic peptides used for monoclonal antibody (mAb) production and assay development were purchased from the Chinese Peptide Company (Beijing, China).

\subsection{In vitro cleavage}

Purified type III collagen from human placenta (Abcam, Cambridge, UK) was cleaved with pro-ADAMTS1,-4, and -8 and MMP-9 (Calbiochem, Merck, Whitehouse Station, NJ, USA). $50 \mu$ g pro-ADAMTS' or MMP-9 was activated with $20 \mu \mathrm{L} 1 \mathrm{mM}$ 4-aminophenylmercuric acetate (APMA) in dimethyl sulfoxide 
(DMSO) and incubated at $37^{\circ} \mathrm{C}$ for 2 hours. $1 \mathrm{mg} / \mathrm{mL}$ type III collagen, diluted in $0.5 \mathrm{M}$ acetic acid, was dialyzed into MMP buffer (100 mM Tris- $\mathrm{HCl}, 100 \mathrm{mM} \mathrm{NaCl}, 10 \mathrm{mM} \mathrm{CaCl}, 2 \mathrm{mM} \mathrm{Zn}$ acetate, $\mathrm{pH}$ 8.0) for two days at $4^{\circ} \mathrm{C}$, and proteins below $10 \mathrm{kDa}$ removed by filter centrifugation (Microcon Ultracel YM-10, cat. no. 42407, Millipore, Billerica, MA, USA). Each digest was performed separately by mixing $100 \mu \mathrm{g}$ type III collagen with $1 \mu \mathrm{g}$ of enzyme (ADAM-TS $-1,-4,-8$ or MMP-9) in MMP buffer $(n=4)$ ). As a control, $100 \mu \mathrm{g}$ of type III collagen was mixed with MMP buffer only. All digests were incubated for $24 \mathrm{~h}$ at $37^{\circ} \mathrm{C}$ and terminated using $5 \mathrm{mM}$ EDTA. Cleavage with cathapsin $\mathrm{K}$ and $\mathrm{S}$ was done in $25 \mathrm{mM} \mathrm{NA}_{2} \mathrm{HPO}_{4}, 150$ $\mathrm{mM} \mathrm{NaCl}, 2 \mathrm{mM}$ EDTA, $2 \mathrm{mM}$ DTT, pH 6.5 at $37^{\circ} \mathrm{C}$ and the digest was stopped after $24 \mathrm{~h} 1 \mu \mathrm{M}$ cysteine protease inhibitor E64 (cat.no.219377, Calbiochem, Merck, Whitehouse Station, NJ, USA). Collagen cleavage was verified by SDS-PAGE separation, and visualized using SilverXpress ${ }^{\circledR}$ Silver Staining Kit (cat. no. LC6100, Invitrogen, Carlsbad, Ca, USA) according to the manufacturer's instructions.

\subsection{Peptide identification}

Peptide fragments of in vitro cleaved type III collagen were identified using liquid chromatography coupled to electrospray ionization (ESI) tandem mass spectrometry (LC-MS/MS). Type III collagen/ ADAMTS digests were ultra-filtrated to remove proteins above $10 \mathrm{kDa}$, the $\mathrm{pH}$ adjusted to 2.0 using formic acid and $4 \mu \mathrm{L}$ of sample was analyzed by LC-MS/MS. LC was performed on a nanoACQUITY UPLC BEH C18 column (Waters, Milford, MA, USA) using a formic acid/acetonitrile gradient. MS and MS/MS were performed on a Synapt High Definition Mass Spectrometry quadruple time of flight MS (QUADTOF; Waters, Milford, MA, USA), with an acquisition range of $350-1600 \mathrm{~m} / \mathrm{z}$ in MS and 50-2000 m/z, in MS/MS. The software "ProteinLynx Global SERVER (PLGS)" (Waters, Milford, MA, USA) was used to analyze spectra and generate peak lists. To identify peptides, MS and MS/MS data was searched against a type III collagen (FASTA) protein database using the Mascot 2.2 (Matrix Science, Boston, MA, USA) software with ESI-QUAD-TOF settings with carbamidomethyl (C), oxidation of methionine (M), oxidation of lysine (K) and oxidation of proline (P) as variable modifications. Only sequences identified with a significant Mascot score were regarded further.

\subsection{Selection of peptide for immunizations}

The first six amino acids of collagen fragment free ends, identified by MS, were regarded as a neoepitope generated by each ADAMTS in question. All protease-generated sequences were analyzed for homology and distance to other cleavage sites, then blasted for homology using the NPS@: network 
protein sequence analysis [42]. Six type III collagen sequences, identified in fragments generated by ADAMTS cleavage, were selected for immunizations.

\subsection{Immunization procedure}

Six 4-6 week old Balb/C mice were immunized subcutaneously in the abdomen with $200 \mu \mathrm{L}$ emulsified antigen (50 $\mu \mathrm{g}$ per immunization) per immunization program, using Freund's incomplete adjuvant. Immunizations using the following immunogen provided a neoepitope specific antibody: GAPGFRGPAGCGG-KLH (American Peptide, Hongkong, China). Immunizations were performed at two-week intervals until stable titer levels were obtained. At each bleeding, the serum titer was investigated and the mouse with the highest titer was selected for fusion. The selected mice were boosted intravenously with $50 \mu \mathrm{g}$ immunogen in $100 \mu \mathrm{L} 0.9 \%$ sodium chloride solution three days before isolation of the spleen for cell fusion. Only one immunogen resulted in satisfactory immune response; the remaining programs were terminated.

\subsection{Fusion and antibody screening}

The fusion procedure has been described elsewhere [43]. Briefly, mouse spleen cells were fused with SP2/0 myeloma fusion partner cells. The hybridoma cells were cloned using a semi-solid medium method and transferred into 96-well microtiter plates for further growth. Here, standard limited dilution was used to promote monoclonal growth. Supernatants were screened using an indirect ELISA, while the biotinylated peptide GAPGFRGPAG-K-Biotin (American Peptide, Hongkong, China) was used as a catcher peptide on streptavidin-coated microtitre plates.

\subsection{Characterization of clones}

Native reactivity and peptide binding of mAbs was evaluated in a preliminary competitive ELISA. mAbs in hybridoma supernatant competed with samples of human, rat or mouse serum and plasma, for biotinylated peptide $(5 \mathrm{ng} / \mathrm{mL})$, bound to a streptavidin coated microtitre plate. Clones were also tested against the free synthetic peptide (GAPGFRGPAG), a non-sense synthetic peptide, and a synthetic peptide elongated with one amino acid at the free N-terminal end (RGAPGFRGPAG). Isotyping of the mAbs was performed using the Clonotyping System-HRP kit, cat.5300-05 (Southern Biotech, Birmingham, AL, USA). The selected clones were purified using Protein $G$ columns according to manufacturer's instructions (GE Healthcare Life Science, Little Chalfont, Buckinghamshire, UK). 


\subsection{COL3/ADAMTS ELISA protocol}

We labeled selected mAbs (5D9) with horseradish peroxidase (HRP) using the Lightning link HRP labeling kit according to the instructions of the manufacturer (Innovabioscience, Babraham, Cambridge, UK). A 96-well streptavidin plate was coated with biotinylated synthetic peptide GAPGFRGPAG-K-Biotin dissolved in assay buffer ( $25 \mathrm{mM}$ PBS, 1\% BSA, 0.1\% Tween-20, pH 7.4 adjusted at $4^{\circ} \mathrm{C}$ ) and incubated 30 minutes at $20^{\circ} \mathrm{C} .20 \mu \mathrm{L}$ of free 10 amino acid peptide calibrator (GAPGFRGPAG) or sample were added to appropriate wells, followed by $100 \mu \mathrm{L}$ of HRP conjugated mAb and incubated 1 hour at $20^{\circ} \mathrm{C}$. Finally, 100 $\mu \mathrm{L}$ tetramethylbenzinidine (TMB) (Kem-En-Tec cat.438OH) was added and the plate was incubated for 15 minutes at $20^{\circ} \mathrm{C}$ in the dark. All the above incubation steps included shaking at $300 \mathrm{rpm}$. After each incubation step, the plate was washed five times in washing buffer ( $20 \mathrm{mM}$ Tris, $50 \mathrm{mM} \mathrm{NaCl}, \mathrm{pH}$ 7.2). The TMB reaction was stopped by adding $100 \mu \mathrm{L}$ of stopping solution $(1 \% \mathrm{HCl})$ and measured at $450 \mathrm{~nm}$ with $650 \mathrm{~nm}$ as the reference. A calibration curve was plotted using a 4-parametric mathematical fit model.

\subsection{Technical evaluation}

From 2-fold dilutions of quality control (QC) samples consisting of serum and plasma samples, linearity was calculated as a percentage of recovery of the $100 \%$ sample. The lower limit of detection (LDL) was determined from 21 zero samples (i.e. buffer) and calculated as the mean plus three standard deviations. The inter- and intra-assay variation was determined by 10 independent runs of six QC samples. The developed COL3/ADAMTS ELISA was evaluated using protease digests described under "In vitro cleavage". $20 \mu \mathrm{L}$ from a sample diluted 1:20 was used for the assay.

\subsection{Explant cultures}

Human synovial tissue and cartilage used for ex vivo cultures was collected from OA patients undergoing total knee replacement at Gentofte Hospital, Denmark. All patients gave written informed consent (Danish science ethical committee, amendment no. 53713, approval no. H-D-2007-0084). Synovial tissue and cartilage were stored separately until receipt. Human synovial membrane samples were cleaned of visible fat, and cut into explants (hSMEx) of $30 \pm 5 \mathrm{mg}$. Human cartilage was punched into $5 \mathrm{~mm}$ explants (HEX) with a biopsy punch (Miltex, MTP-33-35). hSMEx were co-cultured with HEX at a one-to-one ratio, or cultured separately as mono-cultures. All explants were cultured in Dulbecco's Modified Eagle 
Medium supplemented with F12, GlutaMAX ${ }^{\mathrm{TM}}$ and $1 \%$ penicillin and streptomycin (Sigma-Aldrich, Denmark) in 48 well plates for 14 days; without treatment (W/O) or with TNF $[20 \mathrm{ng} / \mathrm{mL}]$ and oncostatin $\mathrm{M}(\mathrm{OSM})[10 \mathrm{ng} / \mathrm{mL}](\mathrm{O}+\mathrm{T})$. Conditioned media was removed and fresh treatment added every 2-3 days. Undiluted conditioned media was used for the measurement of COL3/ADAMTS. Synovial tissue from two patients and cartilage from four patients was used. Synovial tissue and cartilage from the same patient were co-cultured. However due to insufficient amount of cartilage, the synovial tissue was also co-cultured with cartilage from another patient. A total of six replica were included in the experiment.

\subsection{Statistical analysis}

Results are shown as mean with $95 \%$ confidence intervals, SEM or SD. Differences between mean values were compared by either Mann-Whitney's t-test for two-tailed observations or ANCOVA adjusting for covariates (age, gender, BMI and smoker status (yes/no)). The P values were Bonferroni corrected. Associations between the marker COL3/ADAMTS and clinical pain scores were tested weight least squares multiple regression (AutoWeight $1 / \mathrm{SD}^{\wedge} 2$ ) adjusting for the covariates. Odds ratio (OR) and area under the curve (AUC) were calculated by logistic regression including the covariates. P values less than 0.05 were considered significant. Statistical analyses were performed Medcalc ${ }^{\circledR}$ (version 17.2) and plots were done by using GraphPad Prism (version 7).

\section{RESULTS}

\subsection{Identification of ADAMTS generated type III collagen fragments}

A large number of type III collagen fragments with statistically significant Mascot score $(p<0.05)$ were identified after in vitro cleaved by ADAMTS $-1,4$, or -8 . The protease-generated neoepitopes were tested for homology between human and rat. The sequence $486^{\prime} \Downarrow_{\text {GAPGFRGPAG' }} 495$ (C3A) in the alpha 1 chain of type III collagen, generated by ADAMTS $-1,-4$ and $-8(\downarrow)$, was selected for immunizations since it is $100 \%$ homologous to human and rat type III collagen. 


\subsection{COL3/ADAMTS ELISA development, characterization and technical performance}

An IgG1 mAb was produced, selected and used for the development of a solid-phase competitive ELISA, COL3/ADAMTS. The ELISA signal was completely inhibited by $250 \mathrm{ng} / \mathrm{mL}$ of specific neoepitope peptide, whereas an elongated peptide inhibited the signal by less than $<10 \%$ at the same concentration (Fig. 1A). A nonsense peptide did not inhibit the signal (Fig. 1A). Type III collagen cleaved by different ECM associated proteases was tested in the COL3/ADAMTS ELISA to verify the sensitivity towards the target epitope. It was observed that ADAMTS $-1,-4$, and -8 were able to generate the COL3/ADAMTS fragment (Fig. 1B), with ADAMTS-4 generating by far the most. There was little or no reactivity towards intact human type III collagen or fragments generated by the cathepsins and MMPs tested. These findings were consistent in two repeated cleaved batches.

The assay specifications are summarized in Table 2 . Briefly, the assay range was between 2 and $60 \mathrm{ng} / \mathrm{ml}$ and linearity was within $100 \pm 15 \%$ for human serum down to an 8 -fold dilution (table 2 ). The inter-and intra-assay variations, using human serum and urine QC samples, were both below $10 \%$ (table 2).

\subsection{Biological target validation}

COL3/ADAMTS was measured in the conditioned media of human osteoarthritic cartilage explants (HEXs), synovial membrane explants (hSMEx), and co-cultured explants, over the course of 14 days using the COL3/ADAMTS ELISA. COL3/ADAMTS was released from co-cultures, and hSMExs alone, with O+T stimulation in a time-dependent fashion, with peak release at day 3 (Fig. 2A). Patient associated variance was observed, especially with hSMExs, however release of COL3/ADAMTS was 3-fold higher from co-cultured explants after 14 days of $0+T$ stimulation, compared to unstimulated controls (w/o), ( $<<0.05$, Fig. 2B) . 


\subsection{Association between serum COL3/ADAMTS and patient reported pain in $\mathrm{OA}$}

The average concentration of COL3/ADAMTS in serum collected from 261 subjects with or without knee pain was 21.8 [19.4 to 24.0 ] ng/ml. Serum COL3/ADAMTS levels were weakly, but significantly, inversely associated with pain; determined by VAS $(r=-0.15, p<0.05)$, WOMAC $(r=-0.17, p<0.01)$ and Lequesne $(r=-$ $0.13, p<0.05$ ) assessment, following adjustment for age, BMI and gender (Table 3). There was no association between COL3/ADAMTS levels and WOMAC function or stiffness. There was no linear correlation between KL grade and COL3/ADAMTS ( $p_{\text {ANOVAA }}>0.1$, data not shown).

Subjects were separated into four sub-categories based on their VAS pain level, followed by separation into those with or without radiographic knee OA (RKOA) (Table 4). There was a significant difference in serum levels of COL3/ADAMTS between subjects with no pain, and subjects with mild $(p=0.0076)$ or moderate pain ( $p=0.0019)$, when RKOA was not considered. Within the RKOA group, COL3/ADAMTS was significantly elevated in patients with mild pain $(p=0.0086)$ but there was no difference between nonRKOA and RKOA patients; thus COL3/ADAMTS levels were not associated with radiographic knee OA, but rather with pain status.

Next, the performance of COL3/ADAMTS was compared to other markers of ECM degradation and joint pathology, which have previously been published [18][21]. COL3/ADAMTS could significantly predict who would had symptomatic knee OA versus those without (Table 5), with an odds ratio of 0.56 demonstrating a significant inverse relationship $(p=0.0003)$. Another marker of type III collagen degradation, C3M, which is derived from increased metalloproteinase activity, also inversely correlated with symptomatic RKOA and performed better than COL3-ADAMTS; AUC of 0.78 vs 0.72 (Table 5). An ADAMTS derived fragment, AGNx1, a metabolite of aggrecan, positively correlated with symptomatic RKOA but performed worse than COL3-ADAMTS; 0.65 vs. 0.72 . COL3/ADAMTS performed better than age and BMI alone (Table 5). 


\section{DISCUSSION}

In this work, we present the identification and development of a novel ELISA-based marker, which measures human type III collagen cleavage derived from the activity of ADAMTS. The work showed that a sensitive monoclonal antibody was produced, which specifically recognized the collagen type III neoepitope, COL3/ADAMTS, and not an elongated or nonsense peptide. The assay was technically robust and sufficiently sensitive to detect COL3/ADAMTS in patient serum. We also found that the neoepitope was released from human OA synovial membrane when culture in the presence of proinflammatory cytokines, indicating that the marker was indeed associated with joint tissue turnover and could be relevant as a marker for joint health.

\subsection{The association between COL3/ADAMTS and OA pain}

We tested the ELISA in a cross-sectional cohort, which included 261 subjects with varying degree of symptomatic and radiographic OA. We found that serum levels of COL3/ADAMTS significantly and inversely correlated with patient pain score $(r \approx-0.15)$. Moreover, that this association was found to be related to symptomatic radiographic $O A$ rather than knee pain alone (non-radiographic OA). The correlation was weak $(r<0.2)$ which indicates that COL3/ADAMTS is not a strong surrogate marker of pain severity. However, it may provide insight to the underlining pathogenesis of ECM remodeling in OA and its association with development of pain, which is a key diagnostic feature in OA [22]. Lastly, we compared COL3/ADAMTS with other synovial or cartilage markers of either type III collagen (C3M [23]) or aggrecanase activity (AGNx1 [24]). We found that low level of COL3/ADAMTS was predictive of symptomatic radiographic $\mathrm{OA}$ with an odds ratio of $1.8(1 / 0.56, \mathrm{p}=0.0003$, table 5$)$. This was slightly better than high level of AGNx1 which had an odds ratio of 1.8 , but borderline significant $(p=0.066)$, but not as significant as low C3M, which predicted with an odds ratio of $2.5(1 / 0.40, p<0.0001)$.

Pain in $\mathrm{OA}$ is considered multimodal and this weak correlation may indicate that type III collagen degradation reflects only part of the underlining mechanism observed in knee pain. Interestingly, the correlation was independent of radiographic disease severity, which further supports the idea that radiographic $O A$ and pain in $O A$ are not strictly associated [22]. Our results point to the possibility that changes in the turnover, both elevated and decreased, of joint connective tissue is associated with increased structural severity of OA, which cannot be described by pain or demographics alone. We 
speculate that COL3/ADAMTS is a potential biomarker for the identification of patients with minimal symptomatic knee $O A$, but with early and potentially progressing $O A$.

The pain intensity reported by OA patients seems to be only weakly correlated with radiological score, suggesting factors other than cartilage destruction and bony changes are important in OA pain [25]. Recent studies have investigated specific groups of patients with $O A$, characterized by high pain intensities but low radiological severity, and these patients seems to be highly sensitive to pain $[16,26]$. Further, low radiological severity before total knee replacement (TKR) has been linked to low functionality after surgery and recently, low radiological scores in combination with pain sensitivity profiles were found associated with less pain relief following TKR [27]. The current study found COL3/ADAMTS to be associated with pain but not radiological scores, which further supports that radiological $O A$, might be less important compared with clinical pain.

\subsection{COL3/ADAMTS and its association with joint biology}

The synovial membrane is remodeled during the pathogenesis of $\mathrm{OA}$, driven by pro-inflammatory cytokines; and by fragments known as DAMPs (damage associated molecular patterns) which are released from cartilage into the joint cavity upon trauma, and stimulate toll-like receptors which mediate further inflammation [28]. As synovial fibroblasts are activated, they proliferate and start secreting ECM proteins such as type III collagen. The synovial membrane thickens and become vascularized. OA pain is derived from many tissue components, but synovial vascularization and innervation are believed to be major contributors [21,29]. Increased expression and turnover of type III collagen, with associated release of COL3/ADAMTS from a thickening synovial membrane, may precede the onset of mild to moderate pain, when the tissue becomes innervated. Indeed, OA patients with no pain had higher levels of serum COL3/ADAMTS compared to those with mild to moderate pain. In addition, hSMEx COL3/ADAMTS release peaked with inflammatory stimulation early in the explant model at day 3, and decreased to W/O control levels by day 14. Further indicating that COL3/ADAMTS is a marker, which may flag the transition between asymptomatic OA and early progressive symptomatic OA.

ADAMTS expression has been shown to be elevated in synoviocytes and chondrocytes of OA patients $[30,31]$, and plays a central role in the remodeling of joint connective tissues. Several markers and fragments of aggrecan resulting from ADAMTS activity have been described. Fragments containing the 
sequences ARGS and NITEGE (e.g. measured by the AGNx1 ELISA) have been extensively investigated, and biomarkers measuring these neoepitopes have been developed [32,33]. Previously, ADAMTS activity was associated only with degradation and deterioration of the joint; however, recent data suggest that ADAMTS' play a vital role in overall tissue turnover. Elevated ADAMTS expression may therefore reflect an ongoing, potentially uncontrolled, repair process in response to inflammation and injury; and may explain the inverse relationship between COL3/ADAMTS and pain observed in this study.

\subsection{The perspective}

At present there no DMOAD available for OA, however drug are being developed which are believe to change joint metabolism. One of these treatments are targeting ADAMTS [34], which have traditionally been monitored by biomarkers reflecting cartilage degradation through the release of the neo-epitopes ARGS or TEGE from aggrecan [35]. COL3/ADAMTS could be used as a pharmacodynamics marker measuring the effect of anti-ADAMTS' on other tissues than cartilage.

\subsection{Limitations.}

There are several limitations in this study. Firstly, patients with systemic diseases other than musculoskeletal disease were not excluded from the study and results were not adjusted for NSAID use; both of which could influence serum biomarker levels. Secondly, the clinical study investigated a crosssectional cohort, which focused on pain parameters alone, thus cannot provide insight in to the prognostic value of the biomarker.

\section{CONCLUSION}

In conclusion, the COL3/ADAMTS ELISA was technically robust and specific for the selected ADAMTS-1, 4-, -8 generated neoepitope. There was an inverse correlation between COL3/ADAMTS and pain scores, which was mainly associated with radiographic OA. These data may indicate that COL3/ADAMTS is a marker of early osteoarthritis. Such a biomarker could be used to characterize patients with early and mildly progressed disease, and may shed light on which degenerative components contribute to pain perception; since there is a disconnect between the degree of joint degeneration and joint pain. 


\section{ACKNOWLEDGEMENT}

We acknowledge the funding from the Danish "Ministry of Science. Technology and Science", and the DBoard project which has received funding from the European Union's Seventh Framework Program for research technological development and demonstration under grant agreement No. 305815. We would also like to thank our technician Dorthe Vang and our colleagues at Nordic Bioscience Beijing for their contribution in developing the monoclonal antibody and assay development. As well as our colleagues at the Danish Technical University of Denmark for lending MS equipment.

\section{FORMATTING OF FUNDING SOURCES}

We acknowledge the funding from the Danish "Ministry of Science. Technology and Science", and the DBoard project which has received funding from the European Union's Seventh Framework Program for research technological development and demonstration under grant agreement No. 305815. DJL, MAK and ACBJ are full-time employees of Nordic Bioscience. MAK and ACBJ holds stocks in Nordic Bioscience. 


\section{REFERENCES}

[1] A. Struglics, S. Larsson, M.A. Pratta, S. Kumar, M.W. Lark, L.S. Lohmander, Human osteoarthritis synovial fluid and joint cartilage contain both aggrecanase- and matrix metalloproteinasegenerated aggrecan fragments, Osteoarthr. Cartil. 14 (2006) 101-113.

[2] B.L. Tang, ADAMTS: a novel family of extracellular matrix proteases, Int J Biochem Cell Biol. 33 (2001) 33-44.

[3] L. Troeberg, H. Nagase, Proteases involved in cartilage matrix degradation in osteoarthritis., Biochim. Biophys. Acta. 1824 (2012) 133-45. doi:10.1016/j.bbapap.2011.06.020.

[4] S. Larsson, L.S. Lohmander, A. Struglics, An ARGS-aggrecan assay for analysis in blood and synovial fluid, Osteoarthr. Cartil. 22 (2014) 242-249. doi:10.1016/j.joca.2013.12.010.

[5] E. Gineyts, J.A. Mo, A. Ko, D.B. Henriksen, S.P. Curtis, B.J. Gertz, P. Garnero, P.D. Delmas, Effects of ibuprofen on molecular markers of cartilage and synovium turnover in patients with knee osteoarthritis, Ann.Rheum Dis. 63 (2004) 857-861.

[6] E.G. Canty, K.E. Kadler, Collagen fibril biosynthesis in tendon: a review and recent insights., Comp. Biochem. Physiol. A. Mol. Integr. Physiol. 133 (2002) 979-85. http://www.ncbi.nlm.nih.gov/pubmed/12485687 (accessed December 2, 2016).

[7] J.-J. Wu, M.A. Weis, L.S. Kim, D.R. Eyre, Type III collagen, a fibril network modifier in articular cartilage., J. Biol. Chem. 285 (2010) 18537-44. doi:10.1074/jbc.M110.112904.

[8] C. Kjelgaard-Petersen, A.S. Siebuhr, T. Christiansen, C. Ladel, M. Karsdal, A.-C. Bay-Jensen, Synovitis biomarkers: ex vivo characterization of three biomarkers for identification of inflammatory osteoarthritis., $\quad$ Biomarkers. $20 \quad$ 547-56. doi:10.3109/1354750X.2015.1105497. 
[9] S. Hosseininia, M.A. Weis, J. Rai, L. Kim, S. Funk, L.E. Dahlberg, D.R. Eyre, Evidence for enhanced collagen type III deposition focally in the territorial matrix of osteoarthritic hip articular cartilage., Osteoarthritis Cartilage. 24 (2016) 1029-35. doi:10.1016/j.joca.2016.01.001.

[10] M.A. Karsdal, S.H. Madsen, C. Christiansen, K. Henriksen, A.J. Fosang, B.C. Sondergaard, Cartilage degradation is fully reversible in the presence of aggrecanase but not matrix metalloproteinase activity, Arthritis Res.Ther. 10 (2008) R63.

[11] P. Verma, K. Dalal, ADAMTS-4 and ADAMTS-5: key enzymes in osteoarthritis., J. Cell. Biochem. 112 (2011) 3507-14. doi:10.1002/jcb.23298.

[12] M.A. Karsdal, E. Delvin, C. Christiansen, Protein fingerprints - relying on and understanding the information of serological protein measurements, Clin.Biochem. 44 (2011) 1278-1279.

[13] M.J. Janusz, C.B. Little, L.E. King, E.B. Hookfin, K.K. Brown, S.A. Heitmeyer, B. Caterson, A.R. Poole, Y.O. Taiwo, Detection of aggrecanase- and MMP-generated catabolic neoepitopes in the rat iodoacetate model of cartilage degeneration, Osteoarthr. Cartil. 12 (2004) 720-728.

[14] M.A.A. Karsdal, K. Henriksen, D.J.J. Leeming, P. Mitchell, K. Duffin, N. Barascuk, L. Klickstein, P. Aggarwal, O. Nemirovskiy, I. Byrjalsen, P. Qvist, A.C.C. Bay-Jensen, E.B.B. Dam, S.H.H. Madsen, C. Christiansen, Biochemical markers and the FDA Critical Path: how biomarkers may contribute to the understanding of pathophysiology and provide unique and necessary tools for drug development, Biomarkers. 14 (2009) 181-202. doi:10.1080/13547500902777608.

[15] A.-C.C. Bay-Jensen, Q. Liu, I. Byrjalsen, Y. Li, J. Wang, C. Pedersen, D.J. Leeming, E.B. Dam, Q. Zheng, P. Qvist, M. a. Karsdal, Enzyme-linked immunosorbent assay (ELISAs) for metalloproteinase derived type II collagen neoepitope, CIIM--increased serum CIIM in subjects with severe radiographic osteoarthritis, Clin.Biochem. $44 \quad$ (2011) 423-429. 
doi:10.1016/j.clinbiochem.2011.01.001.

[16] L. Arendt-Nielsen, T.N. Eskehave, L.L. Egsgaard, K.K. Petersen, T. Graven-Nielsen, H.C. Hoeck, O. Simonsen, A.S. Siebuhr, M. Karsdal, A.C. Bay-Jensen, Association between experimental pain biomarkers and serologic markers in patients with different degrees of painful knee osteoarthritis., Arthritis Rheumatol. (Hoboken, N.J.). 66 (2014) 3317-3326. doi:10.1002/art.38856.

[17] R. Altman, E. Asch, D. Bloch, G. Bole, D. Borenstein, K. Brandt, W. Christy, T.D. Cooke, R. Greenwald, M. Hochberg, ., Development of criteria for the classification and reporting of osteoarthritis. Classification of osteoarthritis of the knee. Diagnostic and Therapeutic Criteria Committee of the American Rheumatism Association, Arthritis Rheum. 29 (1986) 1039-1049. http://www.ncbi.nlm.nih.gov/pubmed/3741515 (accessed July 4, 2017).

[18] a. S. Siebuhr, K.K. Petersen, L. Arendt-Nielsen, L.L. Egsgaard, T. Eskehave, C. Christiansen, O. Simonsen, H.C. Hoeck, M. a. Karsdal, a. C. Bay-Jensen, Identification and characterisation of osteoarthritis patients with inflammation derived tissue turnover, Osteoarthr. Cartil. 22 (2014) 44-50. doi:10.1016/j.joca.2013.10.020.

[19] N. Bellamy, W.W. Buchanan, C.H. Goldsmith, J. Campbell, L.W. Stitt, Validation study of WOMAC: a health status instrument for measuring clinically important patient relevant outcomes to antirheumatic drug therapy in patients with osteoarthritis of the hip or knee., J. Rheumatol. 15 (1988) 1833-40. http://www.ncbi.nlm.nih.gov/pubmed/3068365 (accessed July 4, 2017).

[20] M.G. Lequesne, C. Mery, M. Samson, P. Gerard, Indexes of severity for osteoarthritis of the hip and knee. Validation--value in comparison with other assessment tests., Scand. J. Rheumatol. Suppl. 65 (1987) 85-9. http://www.ncbi.nlm.nih.gov/pubmed/3479839 (accessed July 4, 2017). 
[21] K.K. Petersen, A.S. Siebuhr, T. Graven-Nielsen, O. Simonsen, M. Boesen, H. Gudbergsen, M. Karsdal, A.C. Bay-Jensen, L. Arendt-Nielsen, Sensitization and Serological Biomarkers in Knee Osteoarthritis Patients With Different Degrees of Synovitis, Clin. J. Pain. 32 (2015) 1. doi:10.1097/AJP.0000000000000334.

[22] L. Arendt-Nielsen, Joint pain: more to it than just structural damage?, Pain. 158 Suppl 1 (2017) S66-S73. doi:10.1097/j.pain.0000000000000812.

[23] A.C. Bay-Jensen, S. Wichuk, I. Byrjalsen, D.J. Leeming, N. Morency, C. Christiansen, M. a. Karsdal, W.P. Maksymowych, Circulating protein fragments of cartilage and connective tissue degradation are diagnostic and prognostic markers of rheumatoid arthritis and ankylosing spondylitis., PLoS One. 8 (2013) 1-7. doi:10.1371/journal.pone.0054504.

[24] Y. He, Q. Zheng, M. Jiang, S. Sun, T.G. Christiansen, M. Kassem, M. a. Karsdal, A.C. Bay-Jensen, The effect of protease inhibitors on the induction of osteoarthritis-related biomarkers in bovine full-depth cartilage explants., PLoS One. 10 (2015) e0122700. doi:10.1371/journal.pone.0122700.

[25] D.T. Felson, The sources of pain in knee osteoarthritis, Curr.Opin.Rheumatol. 17 (2005) 624-628.

[26] J. Bedson, P.R. Croft, The discordance between clinical and radiographic knee osteoarthritis: a systematic search and summary of the literature, BMC.Musculoskelet.Disord. 9 (2008) 116.

[27] A. Riis, M.S. Rathleff, M.B. Jensen, O. Simonsen, Low grading of the severity of knee osteoarthritis pre-operatively is associated with a lower functional level after total knee replacement: a prospective cohort study with 12 months' follow-up, Bone Joint J. 96-B (2014) 1498-1502. doi:10.1302/0301-620X.96B11.33726.

[28] J. Sokolove, C.M. Lepus, Role of inflammation in the pathogenesis of osteoarthritis: latest findings and interpretations., Ther. Adv. Musculoskelet. Dis. 5 (2013) 77-94. 
doi:10.1177/1759720X12467868.

[29] A. Mathiessen, P.G. Conaghan, Synovitis in osteoarthritis: current understanding with therapeutic implications, Arthritis Res. Ther. 19 (2017) 18. doi:10.1186/s13075-017-1229-9.

[30] S. Roberts, H. Evans, K. Wright, L. van Niekerk, B. Caterson, J.B. Richardson, K.H.S. Kumar, J.H. Kuiper, ADAMTS-4 activity in synovial fluid as a biomarker of inflammation and effusion., Osteoarthr. Cartil. 23 (2015) 1622-6. doi:10.1016/j.joca.2015.05.006.

[31] E. Zhang, X. Yan, M. Zhang, X. Chang, Z. Bai, Y. He, Z. Yuan, Aggrecanases in the human synovial fluid at different stages of osteoarthritis., Clin. Rheumatol. 32 (2013) 797-803. doi:10.1007/s10067-013-2171-0.

[32] F.M. Germaschewski, C.J. Matheny, J. Larkin, F. Liu, L.R. Thomas, J.S. Saunders, K. Sully, C. Whittall, Y. Boyle, G. Peters, N.M. Graham, Quantitation OF ARGS aggrecan fragments in synovial fluid, serum and urine from osteoarthritis patients., Osteoarthritis Cartilage. 22 (2014) 690-7. doi:10.1016/j.joca.2014.02.930.

[33] M.G. Chambers, L. Cox, L. Chong, N. Suri, P. Cover, M.T. Bayliss, R.M. Mason, Matrix metalloproteinases and aggrecanases cleave aggrecan in different zones of normal cartilage but colocalize in the development of osteoarthritic lesions in STR/ort mice, Arthritis Rheum. 44 (2001) 1455-1465.

[34] M.A. Karsdal, M. Michaelis, C. Ladel, A.S. Siebuhr, A.R. Bihlet, J.R. Andersen, H. Guehring, C. Christiansen, A.C. Bay-Jensen, V.B. Kraus, Disease-modifying treatments for osteoarthritis (DMOADs) of the knee and hip: lessons learned from failures and opportunities for the future., Osteoarthr. Cartil. 24 (2016) 2013-2021. doi:10.1016/j.joca.2016.07.017.

[35] M.Z. Ilic, C.J. Handley, H.C. Robinson, M.T. Mok, Mechanism of catabolism of aggrecan by 
articular cartilage, Arch Biochem Biophys. 294 (1992) 115-122. 
Table 1. The C4P-003 study overview

\begin{tabular}{|c|c|c|c|c|c|c|c|c|}
\hline \multirow[b]{2}{*}{$\mathbf{N}$} & \multicolumn{2}{|c|}{$\begin{array}{l}1 \text { (No pain) } \\
\text { VAS pain <10 } \\
\text { mm }\end{array}$} & \multicolumn{2}{|c|}{$\begin{array}{l}2 \text { (mild pain) } \\
\text { VAS pain 10-40 } \\
\mathrm{mm}\end{array}$} & \multicolumn{2}{|c|}{$\begin{array}{l}3 \text { (moderate } \\
\text { pain) } \\
\text { VAS pain } 40-70 \\
\mathrm{~mm}\end{array}$} & \multicolumn{2}{|c|}{$\begin{array}{l}4 \text { (severe pain) } \\
\text { VAS pain } 70-100 \\
\text { mm }\end{array}$} \\
\hline & 52 & & 81 & & 67 & & 61 & \\
\hline No. of females (\%) & $23(44)$ & & $46(57)$ & & $35(52)$ & & $34(56)$ & \\
\hline & Mean & SD & Mean & SD & Mean & SD & Mean & SD \\
\hline Age (years) & 67.5 & 4.8 & 62.8 & 8.3 & 64.7 & 7.6 & 63.0 & 8.1 \\
\hline BMI & 27.2 & 4.0 & 27.5 & 3.8 & 27.9 & 3.3 & 29.5 & 4.9 \\
\hline $\begin{array}{l}\text { Disease duration } \\
\text { (years) }\end{array}$ & 6.9 & 4.9 & 6.4 & 5.8 & 8.1 & 12.9 & 9.9 & 1.2 \\
\hline $\begin{array}{l}\text { VAS pain score } \\
(\mathrm{mm})\end{array}$ & 1.1 & 2.3 & 26.2 & 9.3 & 54.3 & 8.1 & 78.7 & 7.0 \\
\hline Lequesne pain score & 0.8 & 1.5 & 4.3 & 1.6 & 5.0 & 1.5 & 5.8 & 1.8 \\
\hline WOMAC_PAIN & 1.0 & 2.0 & 6.6 & 2.8 & 8.1 & 2.5 & 9.4 & 3.1 \\
\hline WOMAC function & 2.3 & 4.1 & 14.8 & 9.1 & 22.9 & 10.6 & 28.0 & 10.5 \\
\hline WOMAC stiffness & 0.6 & 1.1 & 3.0 & 1.5 & 3.9 & 1.3 & 4.4 & 1.6 \\
\hline KLG, worst score & 1.5 & 0.7 & 1.9 & 0.8 & 2.3 & 1.1 & 2.2 & 0.9 \\
\hline
\end{tabular}


Table 2: COL3/ADAMTS ELISA technical performance. A: Measurement range. B: Linearity (percentage dilution recovery) of the COL3/ADAMTS ELISA using human serum samples from healthy donors $(n=3)$. C: Inter- and intra-assay variation of the COL3/ADAMTS ELISA using human serum and urine QC samples; $\mathrm{n}=6$ with 10 independent runs of each QC sample.

\begin{tabular}{|c|c|c|c|c|c|c|}
\hline \multicolumn{7}{|c|}{ Technical performance of the COL3/ADAMTS ELISA } \\
\hline \multirow[t]{2}{*}{ A: Measurement range } & \multirow{2}{*}{\multicolumn{2}{|c|}{$13.7[12.7-14.9]$}} & \multirow{2}{*}{\multicolumn{2}{|c|}{\begin{tabular}{|l|l|} 
Slope \\
$0.99[0.90-1.07]$ \\
\end{tabular}}} & \multicolumn{2}{|c|}{$\begin{array}{l}\text { Quantification } \\
\text { range, } n g / m L\end{array}$} \\
\hline & & & & & 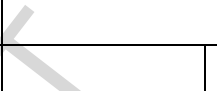 & $2-60$ \\
\hline B: Linearity & $\begin{array}{c}\text { Conc., } \\
n g / m L\end{array}$ & $\begin{array}{r}\% \\
\text { recovery }\end{array}$ & $\begin{array}{c}\text { Conc., } \\
n g / m L\end{array}$ & $\begin{array}{r}\% \\
\text { recovery }\end{array}$ & $\begin{array}{c}\text { Conc., } \\
n g / m L \\
\end{array}$ & $\begin{array}{r}\% \\
\text { recovery }\end{array}$ \\
\hline Undiluted human serum sample & 27.4 & 100.0 & 37.2 & 100.0 & 47.3 & 100.0 \\
\hline Dilution 1 in 2 & 14.0 & 102.4 & 17.9 & 96.3 & 24.9 & 105.3 \\
\hline Dilution 1 in 4 & 7.3 & 106.8 & 8.4 & 90.6 & 13.1 & 110.4 \\
\hline Dilution 1 in 8 & 3.6 & 105.5 & 4.5 & 96.4 & 6.0 & 101.6 \\
\hline Dilution 1 in 16 & 2.0 & 118.6 & 2.6 & 112.1 & 3.7 & 124.3 \\
\hline Average recovery, $\%$ & & 99.1 & & 106.6 & & 108.3 \\
\hline C: Assay Variability & & 8 & $\longrightarrow$ & & & \\
\hline Precision & $n g / m L$ & & $\begin{array}{l}\text { Intra-asso } \\
\text { variability }\end{array}$ & & $\begin{array}{l}\text { Inter-assay } \\
\%\end{array}$ & variability \\
\hline Sample 1 & & 31.6 & & 5.5 & & 7.5 \\
\hline Sample 2 & & 50.3 & & 3.6 & & 3.1 \\
\hline Sample 3 & & 60.1 & & 5.7 & & 3.0 \\
\hline Sample 4 & & 69.2 & & 7.0 & & 5.1 \\
\hline Sample 5 & & 64.3 & & 3.9 & & 5.6 \\
\hline Sample 6 & 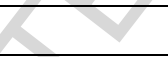 & 80.6 & & 6.0 & & 5.9 \\
\hline Mean & 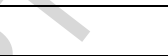 & & & 5.3 & & 5.0 \\
\hline
\end{tabular}


Table 3. Correlation between COL3/ADAMTS and clinical pain and function measures.

\begin{tabular}{|l|l|l|l|l|l|}
\hline & \multicolumn{2}{l|}{ COL3-ADAMTS } & \multicolumn{2}{l|}{$\begin{array}{l}\text { COL3/ADAMTS adjusted for age, gender, BMI } \\
\text { and smoker status }\end{array}$} \\
& R partial & $P$ value & R partial & $P$ value & $\begin{array}{l}\text { Significant } \\
\text { covariates }\end{array}$ \\
\hline VAS PAIN & -0.14 & 0.020 & -0.15 & 0.018 & $\mathrm{BMI}$ \\
\hline WOMAC PAIN & -0.16 & 0.010 & -0.17 & 0.0065 & $\mathrm{BMI}$ \\
\hline Lequesne Pain & -0.17 & 0.0070 & -0.13 & 0.035 & $\begin{array}{l}\text { BMI, Age, } \\
\text { Gender }\end{array}$ \\
\hline WOMAC function & -0.10 & 0.099 & -0.11 & 0.066 & $\mathrm{BMI}$ \\
\hline WOMAC stiffness & -0.08 & $\mathrm{Ns}$ & - & - & $\mathrm{BMI}$, gender \\
\hline
\end{tabular}

Table 4. Levels of serum COL3/ADAMTS in patients with different knee OA pain and radiographic phenotypes. The data was corrected for age, gender, BMI and smoker status.

\begin{tabular}{|c|c|c|c|c|c|c|c|c|c|c|c|c|c|c|c|}
\hline & \multicolumn{3}{|c|}{$\begin{array}{l}1 \text { (No pain) } \\
\text { VAS pain }<10 \mathrm{~mm}\end{array}$} & \multicolumn{4}{|c|}{$\begin{array}{l}2 \text { (mild pain) } \\
\text { VAS pain } 10-40 \mathrm{~mm}\end{array}$} & \multicolumn{4}{|c|}{$\begin{array}{l}3 \text { (moderate pain) } \\
\text { VAS pain } 40-70 \mathrm{~mm}\end{array}$} & \multicolumn{4}{|c|}{$\begin{array}{l}4 \text { (severe pain) } \\
\text { VAS pain } 70-100 \mathrm{~mm}\end{array}$} \\
\hline & $\mathrm{N}$ & Mean & $95 \% \mathrm{Cl}$ & $\mathrm{N}$ & Mean & $95 \% \mathrm{Cl}$ & $P$ & $\mathrm{~N}$ & Mean & $95 \% \mathrm{Cl}$ & & $\mathrm{N}$ & Mean & $95 \% \mathrm{Cl}$ & \\
\hline All & 52 & 31.1 & $\begin{array}{l}25.9 \text { to } \\
36.3\end{array}$ & 81 & 19.2 & $\begin{array}{l}14.4 \text { to } \\
24.0\end{array}$ & 0.0076 & 67 & 17.0 & $\begin{array}{l}11.5 \text { to } \\
22.6\end{array}$ & 0.0019 & 61 & 24.9 & $\begin{array}{l}17.7 \text { to } \\
32.1\end{array}$ & Ns \\
\hline $\begin{array}{l}\text { NON } \\
\text { RKOA }\end{array}$ & 25 & 30.7 & $\begin{array}{l}23.2 \text { to } \\
38.2\end{array}$ & 21 & 20.7 & $\begin{array}{l}12.3 \text { to } \\
29.0\end{array}$ & Ns & 14 & 13.6 & $\begin{array}{l}3.7 \text { to } \\
23.5 \\
\end{array}$ & Ns & 8 & 29.4 & $\begin{array}{l}15.9 \text { to } \\
42.9\end{array}$ & Ns \\
\hline RKOA & 27 & 31.5 & $\begin{array}{l}24.4 \text { to } \\
38.7\end{array}$ & 60 & 17.7 & $\begin{array}{l}12.9 \text { to } \\
22.4\end{array}$ & 0.0086 & 53 & 20.5 & $\begin{array}{l}15.4 \text { to } \\
25.5\end{array}$ & 0.080 & 53 & 20.4 & $\begin{array}{l}15.3 \text { to } \\
25.6\end{array}$ & 0.097 \\
\hline
\end{tabular}

Table 5. Asymptomatic RKOA vs. symptomatic RKOA. The comparisons were done on Z-transformed marker levels.

\begin{tabular}{|l|l|l|l|l|l|l|l|}
\hline & \multicolumn{4}{l}{ The marker } & \multicolumn{2}{l|}{ The model } \\
\hline & OR & $95 \% \mathrm{Cl}$ & P value & AUC & $95 \% \mathrm{Cl}$ & P value & Covariate \\
\hline COL3-ADAMTS & 0.56 & 0.41 to 0.77 & 0.0003 & 0.72 & 0.66 to 0.71 & $<0.0001$ & Age \\
\hline C3M & 0.40 & 0.87 to 0.97 & $<0.0001$ & 0.78 & 0.72 to 0.83 & $<0.0001$ & Age, BMI \\
\hline AGNx1 & 1.84 & 0.96 to 3.53 & 0.066 & 0.65 & 0.59 to 0.71 & 0.024 & Age \\
\hline Age & 0.92 & 0.88 to 0.97 & 0.0010 & 0.67 & 0.61 to 0.73 & 0.0003 & - \\
\hline BMI & 1.08 & 0.99 to 1.16 & 0.093 & 0.59 & 0.53 to 0.65 & 0.083 & - \\
\hline
\end{tabular}


A

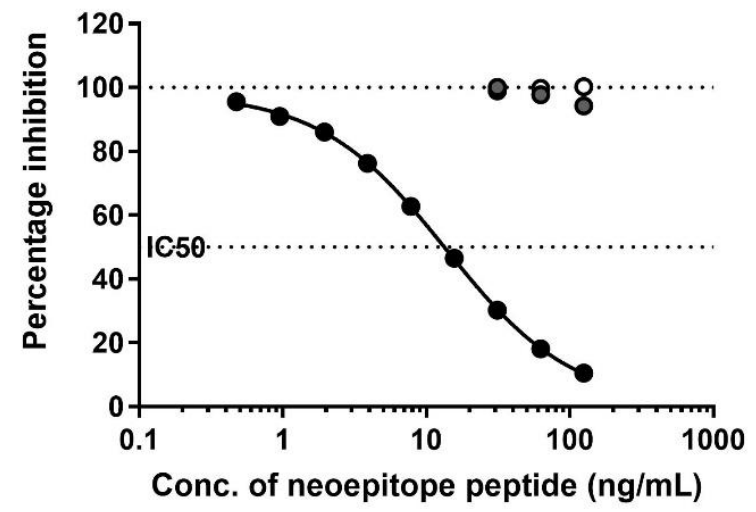

COL3/ADAMTS neoepitope peptide
B

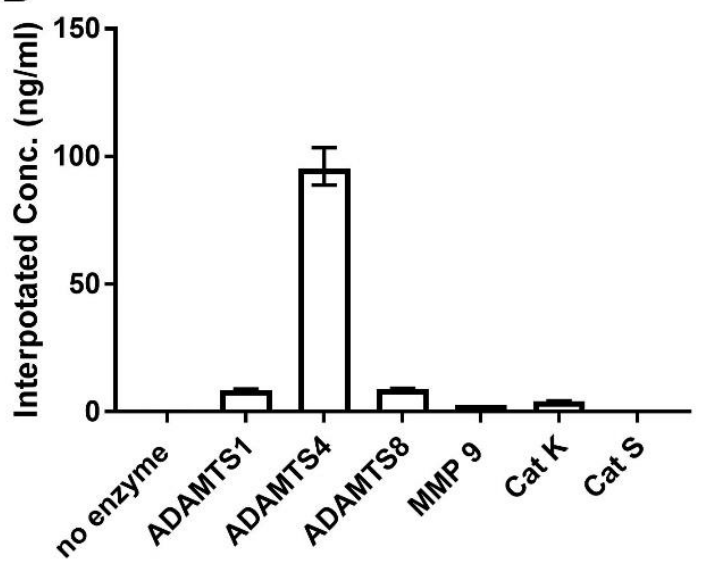

- Elongated elongated peptide 0 Nonsense peptide

Figure 1. Characterization of the COL3/ADAMTS mAb; specificity of type III collagen neoepitope recognition. A) COL3/ADAMTS ELISA standard curve and epitope specificity test. Four-parameter interpolated curve using the specific peptide, as well as test of the elongated and a nonsense peptide. B) COL3/ADAMTS ELISA assessment of in vitro cleaved type III collagen by common proteases of the ECM. Each bar contains four replicates and are shown as mean with SEM bars. 

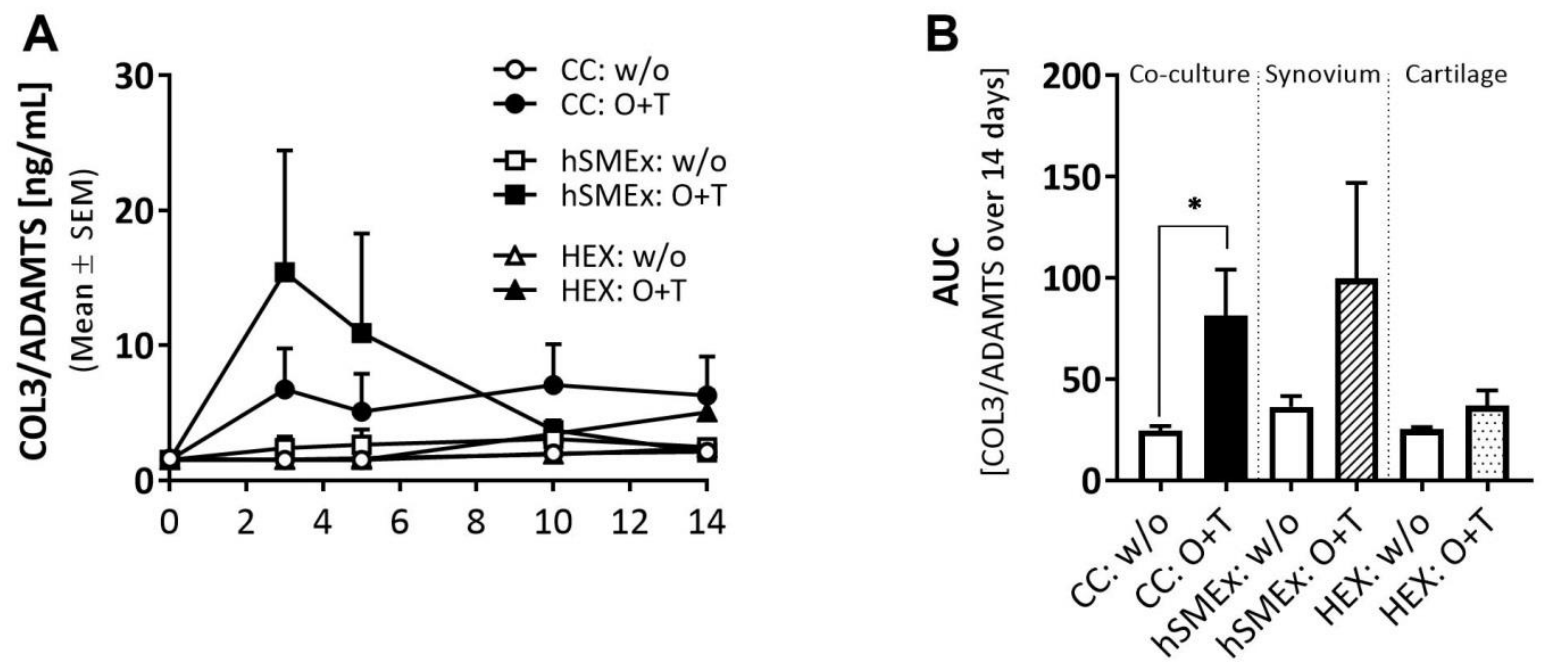

Figure 2. Biological target validation using the COL3/ADAMTS ELISA. A) Time-depended release of COL3/ADAMTS from synovial membrane (hSMEx) or cartilage explants (HEX) alone or in co-cultures (CC) in the presence or absence of the pro-inflammatory cytokines oncostatin $\mathrm{M}$ and TNF alpha $(\mathrm{O}+\mathrm{T})$. Tissue from four different patients was used with six replica per condition. B) Accumulated release of COL3/ADAMTS from the explant cultures after 14 days. Error bars are SEM. * indicates statistical significance $(p<0.05)$. 